\title{
Acknowledgment of Ad Hoc Reviewers
}

The Editor, Dr. Steven Jay Lynn, along with his editorial staff, gratefully acknowledges the assistance of the following individuals, who served as ad hoc reviewers for Psychology of Consciousness: Theory, Research, and Practice between October 2017 and October 2018.

Henk Aarts

Muhammad Abid Azam

Robert A. Ackerman

Davis Alan

Mark Alicke

Christopher Allen

Adrian Alsmith

Chris Anderson

Eric Anderson

Marcia Angell

Adrian Angold

A. Vania Apkarian

Alvaro Arce-Ferrer

Anu Asnaani

Yochai Ataria

Lynn Baker-Ward

Frederick Barrett

Eni Becker

Dan Bernstein

Dorthe Berntsen

Derek Besner

Eric Bijleveld

Mark Blagrove

Hartmut Blank

Susan Bluck

Michael Bogenschutz

Lucie Bonneville

Boris Bornemann

Robert Bornstein

Brad Earl Bowins

Bruno Breitmeyer

Lily Brown

Maggie Bruck

Bradley R. Buchsbaum

Nicole Campione-Barr

Chiu-De Chiu

Kalina Christoff

A. D. (Bud) Craig

Ruud Custers

Michael Dambrun Arnaud D'Argembeau
Brett Deacon

Felipe De Brigard

Evi deCock

Jan De Houwer

Peter de Lissa

Paul Dell

Emily Deming*

Dan Denis

Francesco Dentale

K.Andrew DeSoto

Victoria DiSciullo*

Martin Dorahy

Natalie Ebner

Naomi Eisenberger

Miguel Farias

Mary Ann Foley

Sophie Forster

Kieran Fox

R. Chris Fraley

Michael Franklin

Philip Gerrans

Brandon Gibb

Dan Gilbert

Joseph Glicksohn

Bruce Greyson

Roland Griffiths

Steven Haase

Michael Hallquist

Zachariah I. Hamzagic*

Guy Hawkins

Sarah Hayes-Skelton

Alexandre Heeren

Guido Hesselmann

Gregor Hochstetter

Tim Hollins

Steven Hollon

Lorraine Hope

Matt C. Howard

Nathan W. Hudson

Russell Hurlburt
John Ioannidis

Derek Isaacowitz

Itai Ivtzan

Kiera James*

Jutta Joormann

J. Scott Jordan

Michael Kane

A. K. M. Rezaul Karim

Ed Kelly

Markus Kiefer

Sarah Killoren

Joe Kim

Irving Kirsch

Theo A. Klimstra

Hied Klumpp

Anne Krause-Utz

Ethan Kross

Frank Krueger

Sandra L. Ladd

Hakwan Lau

Steve Laureys

Linda J. Levine

Yunn-Wen Lien

Michael Lifshitz

Kristen Lindquist

David Luke

Antoine Lutz

Spencer Lynn

Jennifer MacCormack

Andrew MacLeod

David MarcussonClavertz

Corinna Martarelli

Reed Maxwell

Chris McFerron

Michael T. McKay

Wolf Mehling

Kourken Michaelian

Matthias Michal 
Michael Miller

Sarah Miller

Joanna Moncrief

Ezequiel Morsella

Michael Mrazek

Bryan Myers

Lauri Nummenmaa

Rory O'Connor

Cristina Ottaviani

Morten Overgaard

Adrian Parker

Alejandro Parra

Benjamin Andrew Parris

Lawrence Patihis

Bryan Paton

David Patterson

Alfredo Pereira Jr.

David Pillemer

Giulia Poerio

Eva M. Pomerantz

Yang Qu

Jason Randall
Anne Richards

Serje Robidoux

Dan Robinson

Tomas Ros

Michael Ross

Jeff Rouder

Clay Routledge

Jan Rummel

Eric Ruthruff

Bastiaan Rutjens

Noam Sagiv

Karen Salmon

Vedat Sar

Adriano Schimmenti

Thomas Schmidt

Casey Schofield

Seth Segall

Paul Seli

Anil Seth

Rick Shelton

Shinsuke Shimojo

John Skowronski

Jamilia Sly

Eli Somer

Rex Stanford
Deryn Strange

Tadas Stumbrys

Madalina L. Sucala

Niels Taatgen

Bert Timmermans

Michelle Toews

Lourdes Trammell

Aliona Tsypes*

Nash Unsworth

Heiddis Valdimarsdottir

Jordi Valiverd'u

Dalena van Heugten-van der Kloet

Marieke Van Vugt

Katalin Varga

Frederick Verbruggen

Amanda M. Vicary

Deming Wang

Mark Williams

David Yaden

Claire Zedelius

* Denotes a co-reviewer. 